\title{
Economic Model-Predictive Control Strategies for Aircraft Deep-stall Recovery with Stability Guarantees*
}

\author{
Torbjørn Cunis, ${ }^{1}$ Dominic Liao-McPherson, ${ }^{2}$ Jean-Philippe Condomines, ${ }^{3}$ \\ Laurent Burlion, ${ }^{4}$ and Ilya Kolmanovsky ${ }^{2}$
}

\begin{abstract}
Aircraft upset recovery requires aggressive control actions to handle highly nonlinear aircraft dynamics and critical state and input constraints. Model predictive control is a promising approach for returning the aircraft to the nominal flight envelope, even in the presence of altered dynamics or actuator limits; however, proving stability of such strategies requires careful algebraic or semi-algebraic analysis of both the system and the proposed control scheme, which can be challenging for realistic control systems. This paper develops economic model predictive strategies for recovery of a fixed-wing aircraft from deep-stall. We provide rigorous stability proofs using sum-of-squares programming and compare several economic, nonlinear, and linear model predictive controllers.
\end{abstract}

\section{INTRODUCTION}

Aircraft upset incidents remain a severe cause of fatalities in civil aviation [1] and this has motivated research into upset and loss of control recovery [2-6]. Upset recovery has been approached with various control techniques including adaptive control [7], machine learning [8], and model predictive control (MPC) [9, 10]. MPC, in particular, is promising since it can handle nonlinearities, actuator saturation, and state constraints. It also tends to have a provably large closed-loop region of attraction.

In this paper, we propose a loss of altitude (LOA) minimizing economic model predictive control (EMPC) strategy for deep-stall recovery which we compare to linear and nonlinear tracking type MPC controllers. LOA is an important performance metric for upset recovery maneuvers and it can be exploited to enlarge the operational envelope during and after the maneuver, particularly at low altitudes [11, 12]. The EMPC framework, see, e.g., [13] for an overview, allows for direct minimization of the LOA. We consider a $1.6 \mathrm{~kg}$ fixed-wing unmanned aircraft capable of stable deep-stall descent [14, 15], which allows us to isolate the longitudinal aerodynamics.

\footnotetext{
${ }^{\star}$ Partly supported by ONERA, University of Michigan Advanced Research Computing, and NSF Award No. CMMI 1562209.

${ }^{1} \mathrm{TC}$ was with the Department of Information Processing and Systems, ONERA - The French Aerospace Lab, 31055 Toulouse, France, and the ENAC Drones Research Group; TC is now with the University of Michigan, tcunis@umich.edu.

${ }^{2} \mathrm{DL}$ and IK are with the Department of Aerospace Engineering, University of Michigan, Ann Arbor, Michigan 48109, USA, \{dliaomcp, ilya\}@umich. edu.

${ }^{3} \mathrm{JC}$ is with the Drones Research Group, ENAC, Université de Toulouse, jean-philippe.condomines@enac.fr.

${ }^{4}$ LB is with Rutgers, The State University of New Jersey, New Brunswick, New Jersey, USA, laurent.burlion@rutgers.edu.
}

MPC has been applied to a variety of aerospace systems [16]. In [9], MPC is employed to generate guidance trajectories for recovery of a piloted aircraft from a highpitch upset. EMPC has been extensively studied in the context of process control [13]; however, comparatively few aerospace applications have been reported in the literature [16]. LOA minimizing EMPC was considered in [10] for automatic recovery from a high-bank condition. However, closed-loop stability of the proposed MPC recovery scheme was not proven in either [9] or [10].

Closed-loop stability of an EMPC controller can be guaranteed if the dynamics and stage cost jointly satisfy an appropriate dissipativity condition both with [17] and without [18] terminal constraints. When dissipativity does not hold (or cannot be proven), regularization terms can be added to ensure closed-loop stability [19]. For nonlinear systems proving these conditions is nontrivial. However, advances in semi-definite relaxations [20] have led to a surge of sum-of-squares (SOS) programming techniques [21, 22] which have been used to compute stability certificates for continuous [23, 24] and discrete $[25,26]$ systems as well as proving dissipativity properties $[27,28]$. Further, an SOS-based stability analysis for general MPC strategies is presented in [29]. However, computational issues and the restriction of SOS programming to polynomial functions conflict with the need for accurate aerodynamic models.

Our contributions are fourfold: (i) We design an LOA minimizing EMPC controller using a piecewise polynomial model of the aircraft dynamics that is suitable for both accurate control and application of SOS techniques; (ii) we illustrate how SOS techniques can be applied to systems with piecewise dynamics in order to rigorously prove the stability of our EMPC controller; (iii) we propose an adaptive regularization scheme using SOS to determine minimal regularization gains that ensure dissipativity; (iv) we provide a comparison between linear, nonlinear, and economic MPC for LOA-minimal recovery. In this work, we restrict ourselves to terminal state constraints.

The layout of the paper is as follows: In II, we discuss the upset recovery problem. In III, we recall the theories of EMPC and SOS programming; we then provide the optimal control formulation in IV. In V, we show using SOS programming that the synthesized closed-loop system satisfies the conditions for asymptotic stability. Finally, VI compares different MPC strategies. 
Notation: Real-valued variables and functions are designated in italic, finite sequences in bold, polynomials in Fraktur. $\mathbf{x}[i]$ is the $i$-th element of a sequence $\mathbf{x} . \bar{x}$ is the difference between $x$ and a reference $x^{*} . \mathbb{R}[x]$ is the set of polynomials with real coefficients. For a function $p$ (polynomial $\mathfrak{p}$ ) and $a \in \mathbb{R}$, denote $\Omega_{p \leq a}=\{x \mid p(x) \leq a\}$ and $\mathcal{O}_{p=a}=\{x \mid p(x)=a\}$. Superscripts ${ }^{\text {pre }}$ and ${ }^{\text {post }}$ denote low and high-angle of attack dynamics.

\section{Problem Formulation}

In the context of aviation, the term upset can be used to describe a variety of abnormal situations. In a technical sense, an upset can be understood as an undesired yet often attractive mode of the nonlinear dynamics that shows significantly altered steady-state responses and usually immediately precedes wing stall and departure of the aircraft (e.g., deep-stall flight, gyroscopic spins, or spirals). Input saturation, inversion and tight state constraints make designing recovery approaches challenging.

In this paper, we develop an MPC strategy to minimize loss-of-altitude using only the elevator $\eta$, i.e., thrust $F=0$, in accordance with governmental procedures for manual recovery [30]. LOA is a crucial metric for both collision avoidance and operating envelope recovery post stabilization [11, 12]. We will adopt the convention that the aircraft has recovered when it returns to a stable trim condition within the region of attraction of a nominal flight controller which subsequently reinstates level flight.

We consider only the longitudinal aircraft dynamics, which are given by

$$
\begin{aligned}
m \dot{V} & =F \cos \alpha-\left(\bar{q} C_{\mathrm{D}}(\alpha, \eta)+m g \sin \gamma\right), \\
m V \dot{\gamma} & =F \sin \alpha+\left(\bar{q} C_{\mathrm{L}}(\alpha, \eta)-m g \cos \gamma\right), \\
\dot{\Theta} & =q, \\
I_{y} \dot{q} & =\left(c_{\mathrm{A}} \bar{q} C_{\mathrm{m}}(\alpha, \eta)-C_{\mathrm{M} q} q\right),
\end{aligned}
$$

with airspeed $V$, inclination $\gamma$, pitch rate $q$, angle of attack $\alpha$, pitch angle $\Theta=\gamma+\alpha$, and elevator deflection $\eta$, where $\bar{q}=\frac{1}{2} \varrho S V^{2}$ and $C_{\mathrm{M} q}>0$ is a linear damping parameter. The aircraft's descent rate is then

$$
\dot{z}_{\mathrm{g}}=-V \sin \gamma \text {. }
$$

The aerodynamic coefficients $C_{\mathrm{L}}, C_{\mathrm{D}}, C_{\mathrm{m}}$ are given as continuous piecewise polynomial models

$$
C_{\odot}(\alpha, \eta)= \begin{cases}\mathfrak{C}^{\text {pre }} & \text { if } \alpha \leq \alpha_{0} \\ \mathfrak{C}^{\text {post }} & \text { else; }\end{cases}
$$

with $\alpha_{0}=16.29^{\circ}$ and $\mathfrak{C}^{\text {pre }}, \mathfrak{C}^{\text {post }} \in \mathbb{R}[\alpha, \eta]$. The polynomials and remaining parameters are provided in [31].

The elevator deflection is physically restricted to values between $-60^{\circ}$ to $20^{\circ}$. In this work, we treat the elevator rate $\dot{\eta}$, rather than the deflection, as a control input, the state is thus $x=(V, \gamma, q, \alpha, \eta)$ and the control input is $u=(\dot{\eta}, F)$. The underlying aerodynamic models are defined on the following regions of the state-space $\mathcal{X}=[5 \mathrm{~m} / \mathrm{s} ; 30 \mathrm{~m} / \mathrm{s}] \times\left[-60^{\circ} ;+60^{\circ}\right] \times\left[-150^{\circ} / \mathrm{s} ;+150^{\circ} / \mathrm{s}\right] \times$ $\left[-10^{\circ} ;+75^{\circ}\right] \times\left[-60^{\circ} ;+20^{\circ}\right]$. The control inputs are restricted to $\mathcal{U}=\left[-200^{\circ} / \mathrm{s} ;+200^{\circ} / \mathrm{s}\right] \times \mathbb{R}_{\geq 0} ;$

\section{Methodology}

Consider a nonlinear system which represents (1),

$$
\dot{x}=f(x, u),
$$

subject to the constraints $x \in \mathcal{X} \subseteq \mathbb{R}^{n}, u \in \mathcal{U} \subseteq \mathbb{R}^{m}$, and its discrete time representation

$$
x^{+}=f^{+}(x, u)=x+\tau f(x, u)
$$

with sampling period $\tau>0$. For some $\mathbf{u} \in \mathcal{U}^{N}$ and $x_{1}=x$, write $x_{N+1}=f^{N}(x, \mathbf{u})$; let $\mathcal{Z}=\mathcal{X} \times \mathcal{U}$ and denote the set of trim conditions as $\mathcal{Z}^{\text {trim }}=$ $\left\{(x, u) \in \mathcal{Z} \mid x=f^{+}(x, u)\right\}$.

Definition 1: A set $\mathcal{X}^{\prime} \subset \mathcal{X}$ is called a stable set for $x_{0} \in \mathcal{X}^{\prime}$ and $\kappa: \mathcal{X}^{\prime} \rightarrow \mathcal{U}$ if and only if

$$
\begin{array}{r}
f_{\kappa}^{N}(x) \in \mathcal{X}^{\prime}, \\
\left|x_{0}-f_{\kappa}^{\infty}(x)\right| \rightarrow 0,
\end{array}
$$

with $f_{\kappa}^{+}=f^{+}(\cdot, \kappa(\cdot))$ for all $x \in \mathcal{X}^{\prime}$ and $N>0$. The region of attraction $\mathcal{R}$ then is the largest ${ }^{*}$ stable set.

The stable sets of (4) can be characterized using Lyapunov's stability theory:

Theorem 1: [32] Let $\mathcal{V}(\cdot)$ be positive-definite with $\mathcal{V}(0)=0$ and $\lambda>0$; if

$$
\nabla \mathcal{V} \cdot\left(f_{\kappa}(x)-x^{*}\right)<0
$$

for all $x \neq x^{*}$ with $\mathcal{V}(\bar{x}) \leq \lambda$, then $\Omega_{\mathcal{V} \leq \lambda}$ is a stable set for $f_{\kappa}\left(x^{*}\right)=0 . \triangleleft$

Stability of (4) follows under some mild conditions [33].

\section{A. Model predictive control}

The model-predictive feedback law is defined by the solution of the following OCP:

Given a measured state $x_{0} \in \mathcal{X}$ and target $\left(x_{f}, u_{f}\right) \in$ $\mathcal{Z}^{\text {trim }}$; solve the constrained nonlinear program

$$
\begin{gathered}
\min _{\mathbf{x}, \mathbf{u}} \sum_{i=1}^{N-1} \ell\left(x_{i}, u_{i}\right), \\
x_{i+1}=f^{+}\left(x_{i}, u_{i}\right), \quad i=0, \ldots, N-1, \\
x_{N}=x_{f}, \quad \mathbf{x} \in \mathcal{X}^{N}, \quad \mathbf{u} \in \mathcal{U}^{N}
\end{gathered}
$$

Here, $\ell: \mathcal{Z} \rightarrow \mathbb{R}$ is called the stage cost. The MPC feedback law is then

$$
u(t)=\hat{\mathbf{u}}[1](x(t))
$$

where $(\hat{\mathbf{x}}, \hat{\mathbf{u}})$ is a minimizer of $(8)$ with $x_{0}=x(t)$. The set of recoverable conditions is further defined as

$$
\begin{array}{r}
\mathcal{Z}_{N}=\left\{\left(x_{0}, \mathbf{u}\right) \in \mathcal{X} \times \mathcal{U}^{N} \mid f^{N}\left(x_{0}, \mathbf{u}\right)=x_{f}\right. \text { and } \\
\left.\forall k \leq N . f^{k}\left(x_{0}, \mathbf{u}^{k}\right) \in \mathcal{X}\right\},
\end{array}
$$

where $\mathbf{u}^{k}$ are the first $k$ elements of $\mathbf{u}$, and $\mathcal{X}_{N}$ denotes the projection of $\mathcal{Z}_{N}$ onto $\mathcal{X}$. We assume that [17]:

Assumption 1: $\mathcal{Z}$ is compact, $\ell$ and $f^{+}$are continuous and $\mathcal{X}_{N}$ contains $x_{f}$ in its interior.

${ }^{*}$ In the sense of $\mathcal{X}^{\prime} \subseteq \mathcal{R}$ for any stable set $\mathcal{X}^{\prime}$. 
The following Theorem provides sufficient conditions for closed-loop stability of EMPC.

Theorem 2: [17] Let $\left(x_{f}, u_{f}\right) \in \mathcal{Z}^{\text {trim }}$ satisfy: ${ }^{\dagger}$

1) $f^{+}$is strictly dissipative with respect to (w.r.t.) the supply rate $\varsigma_{\ell}:(x, u) \mapsto \ell(x, u)-\ell\left(x_{f}, u_{f}\right)$;

2) $\ell\left(x_{f}, u_{f}\right) \leq \ell(x, u)$ for all $(x, u) \in \mathcal{Z}^{\text {trim }}$

then $x_{f}$ is an asymptotically stable equilibrium of (5), (9) with region of attraction $\mathcal{X}_{N}$ as defined above. $\triangleleft$ Recall that:

Definition 2: The control system (5) is called strictly dissipative w.r.t. a supply rate $\varsigma: \mathcal{Z} \rightarrow \mathbb{R}$ if and only if there exists a storage function $\Lambda: \mathcal{X} \rightarrow \mathbb{R}$ such that

$$
\Lambda\left(x^{+}\right)-\Lambda(x) \leq-\rho\left(x-x_{f}\right)+\varsigma(x, u)
$$

for a $\rho: \mathcal{X} \rightarrow \mathbb{R}_{\geq 0}$ positive definite de $^{\S}$ and all $(x, u) \in \mathcal{Z}$. Note that unlike conventional MPC, $\ell$ may not necessarily be positive definite around the target equilibrium.

\section{B. Sum-of-squares programming}

We make extensive use of sum-of-squares (SOS) programming to prove dissipativity and to estimate the region of attraction of the nominal controller. A polynomial $\mathfrak{f} \in \mathbb{R}[x]$ is a sum of squares, $\mathfrak{f}=\sum_{i} \mathfrak{f}_{i}^{2}$ with $\left(\mathfrak{f}_{i}\right)_{i} \subset \mathbb{R}[x]$, if and only if $\mathfrak{f}=\mathfrak{z}(x)^{T} Q \mathfrak{z}(x)$, where $Q \succeq 0$ and $\mathfrak{z}$ is a vector of monomials in $x$ [22]; the set of sum-of-squares polynomials is denoted $\Sigma[x]$. This equivalence reduces the problem " $f \in \Sigma[x]$ " to a semi-definite programming problem [20]. Since sum-of-squares polynomials are nonnegative, the following lemma can be proven.

Lemma 1: [23] Let $\mathfrak{f}, \mathfrak{g}_{1}, \ldots, \mathfrak{g}_{k}, \mathfrak{h} \in \mathbb{R}[x]$; we have

$$
\bigcap_{i} \Omega_{\mathfrak{g}_{i} \leq 0} \cap \mathcal{O}_{\mathfrak{h}=0} \subseteq \Omega_{\mathfrak{f} \leq 0}
$$

if $\sum_{i} \mathfrak{s}_{i} \mathfrak{g}_{i}+\mathfrak{p h}-\mathfrak{f} \in \Sigma[x]$ for $\mathfrak{s}_{1}, \ldots, \mathfrak{s}_{k} \in \Sigma[x]$ and $\mathfrak{p} \in$ $\mathbb{R}[x] \cdot \triangleleft$

If the sufficient condition holds, we write $(\mathbf{s}, \mathfrak{h}) \vdash$ $\bigcap_{i} \Omega_{\mathfrak{g}_{i} \leq 0} \cap \mathcal{O}_{\mathfrak{h}=0} \subseteq_{\Sigma} \Omega_{\mathfrak{f} \leq 0}$ with $\mathbf{s}=\left(\mathfrak{s}_{1}, \ldots, \mathfrak{s}_{k}\right)$, saying that $(\mathbf{s}, \mathfrak{h})$ proves the set inclusion.

\section{Controller Design}

In this section, we devise an EMPC strategy for LOA minimal recovery and a corresponding regularization scheme to ensure dissipativity. No thrust is applied during recovery and we consider only the elevator rate as an input, i.e., $u=\dot{\eta}$. In level flight a nominal flight controller $\kappa_{\text {lvl }}$ stabilizes the aircraft. Thus, the nominal trim condition is unattainable for the elevator-only recovery strategy; instead, we choose a target steady-state $\left(x_{f}, u_{f}\right)$ for EMPC that is contained in the interior of the control-invariant nominal stable set, viz.

$$
\begin{aligned}
& x_{f}=\left(10.8 \mathrm{~m} / \mathrm{s},-2.28^{\circ}, 0^{\circ} / \mathrm{s}, 4.15^{\circ},-6.80^{\circ}\right), \\
& u_{f}=0^{\circ} / \mathrm{s},
\end{aligned}
$$

\footnotetext{
${ }^{\dagger}$ Assumption 2 of [17] is fulfilled if $f^{+}$is locally controllable in an open environment of $x_{f}$.

‡We write hereafter "dissipative w.r.t. the cost $\ell$."

$\S \mathrm{A}$ continuous function $\phi$ is said to be positive definite (p.d.) if $\phi(\cdot)>0$ everywhere except at the origin and $\phi(0)=0$.
}

the unique gliding trim condition with minimal descend speed [15]. We want the EMPC controller to minimize the positive loss-of-altitude, $\Delta z_{\mathrm{g}}=z_{\mathrm{g} N}-z_{\mathrm{g} 0}$, which corresponds to the altitude-loss stage cost

$$
\ell_{\Delta}(x)=z_{\mathrm{g}}^{+}-z_{\mathrm{g}}=-\tau V \sin \gamma .
$$

We denote by $\ell_{\Delta}^{*}$ the steady-state loss of altitude, which satisfies $\ell_{\Delta}^{*}=\ell_{\Delta}\left(x_{f}, u_{f}\right) \leq \ell_{\Delta}(x, u)$ for all $(x, u) \in \mathcal{Z}^{\text {trim }}$. Without propulsion, the aircraft descends in steady-state in order to convert potential into kinetic energy and $\ell_{\Delta}^{*}>0$. However, (14) is not positive definite on $\mathcal{Z}$; the loss of altitude becomes negative (i.e., the aircraft ascends) for any positive inclination $\gamma$. To ensure that the dissipativity condition in Theorem 2 holds we add quadratic regularization terms to the stage cost:

$$
\ell_{R}(x, u)=\ell_{\Delta}(x)+\frac{1}{2}\left\|x-x_{f}\right\|_{Q_{x}}^{2}+\frac{1}{2}\left\|u-u_{f}\right\|_{Q_{u}}^{2},
$$

where $Q_{x} \in \mathbb{R}^{n \times n}, Q_{u} \in \mathbb{R}^{m \times m}$ are positive diagonal matrices. In the next section we illustrate how to determine minimal gains $Q_{x}$ and $Q_{u}$ which ensure dissipativity.

We also investigate the performance of a nonlinear tracking MPC controller which uses the stage cost

$$
\ell_{T}(x, u)=\frac{1}{2}\left\|x-x_{f}\right\|_{Q_{x}}^{2}+\frac{1}{2}\left\|u-u_{f}\right\|_{Q_{u}}^{2} ;
$$

where $Q_{x}$ and $Q_{u}$ are positive definite weighting matrices. The stability of the tracking NMPC can be established using [33, Theorem 5.5]. All controllers enforce the box constraints $(x, u) \in \mathcal{X} \times \mathcal{U}$.

\section{ANALYSIS}

As our main result, we use SOS programming to synthesize a suitable storage-candidate function $\mathfrak{L}$ and regularization gains satisfying the conditions of Theorem 2. We further estimate the region of attraction of the nominal level-flight trim condition to ensure that the target steady-state for recovery lies within reach of the nominal flight controller. In order to transform the nonlinear aircraft dynamics model (1)-(2) into a piecewise polynomial form, we replace sine and cosine by their 3rd-order Taylor polynomials, providing sufficient accuracy within the chosen ranges of $\gamma$ and $\alpha$. Likewise, within the stable neighbourhood the inversion $V^{-1}$ is well approximated by a 5th-order Taylor polynomial. The resulting polynomial functions are denoted by $\mathfrak{f}_{V}, \mathfrak{f}_{\gamma}$, etc. and $x^{+}=\mathfrak{f}^{\text {pre }}\left(\dot{x}=\mathfrak{f}^{\text {pre }}\right)$ if $\alpha \leq \alpha_{0}, x^{+}=\mathfrak{f}^{\text {post }}(\dot{x}=$ $\mathfrak{f}^{\text {post }}$ ) else. For tractability, any terms of $\mathfrak{f}^{\text {pre }}, \mathfrak{f}^{\text {post }}$ with degree larger than 5 or coefficients smaller than $10^{-6}$ are removed with negligible loss of accuracy. The cost supply rate is likewise approximated by $\mathfrak{S}_{\ell} \in \mathbb{R}[x, u]$.

\section{A. Dissipativity $\&$ Regularization}

To prove that the EMPC feedback law is stabilizing, the system dynamics must be strictly dissipative w.r.t. the stage cost $\ell$. Proving this condition requires a suitable storage function $\Lambda: \mathcal{X} \rightarrow \mathbb{R}$ satisfying (11). With an analytical search for $\Lambda$ being intractable in general, a polynomial storage function proving dissipativity can 

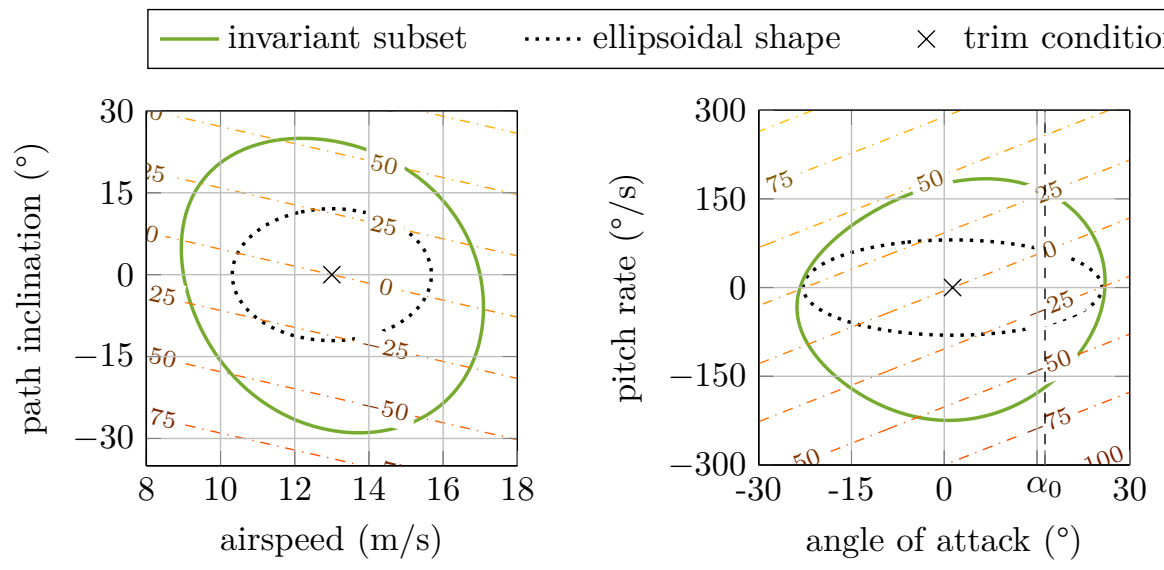

$-0 \cdot-$ actuator rate $(\% / \mathrm{s})$

Fig. 1: Provable stable set under nominal LQR control inputs.

be synthesized by solving a sum-of-squares feasibility problem. However, it is often unknown a-priori if there exists a sum-of-squares polynomial storage function of a given degree which proves dissipativity of the dynamics w.r.t. the chosen stage cost. This can be remedied with a regularization. It has been established in [19] that if the gains $Q=\operatorname{diag}\left(Q_{x}, Q_{u}\right)$ in (15) are chosen sufficiently large, then the dynamics are dissipative w.r.t. the stage cost. There, the authors propose a procedure based on Gershgorin's circle theorem which is computationally simple but prone to conservative (large) regularization gains.

Instead, we propose to use min-trace SOS programming to search simultaneously for a polynomial storagecandidate $\mathfrak{L}$ and diagonal gains $Q$ that directly prove the dissipativity condition (11), by solving the problem

$$
\min _{\substack{Q \succeq 0 \\
\mathbf{s}_{1}, \mathbf{s}_{2} \subset \Sigma[z] \\
\mathcal{L} \in \mathbb{R}[x]}} \operatorname{tr}(Q) \quad \text { s.t. }\left\{\begin{array}{l}
\mathbf{s}_{1} \vdash \mathcal{Z} \cap \Omega_{\alpha \leq \alpha_{0}} \subseteq_{\Sigma} \Omega_{\mathfrak{L}, Q}^{\text {pre }} \\
\mathbf{s}_{2} \vdash \mathcal{Z} \cap \Omega_{\alpha \geq \alpha_{0}} \subseteq_{\Sigma} \Omega_{\mathfrak{L}, Q}^{\text {post }}
\end{array}\right.
$$

where $\Omega_{\mathfrak{L}, Q}^{(\cdot)}$ denotes the set of all $z \in \mathbb{R}^{m+n}$ where

$$
\mathfrak{L} \circ \mathfrak{f}^{(\cdot)}(z)-\mathfrak{L}(z)-\mathfrak{S}_{\ell}(z) \leq \frac{1}{2}\left\|z-z_{f}\right\|_{Q}^{2}-\epsilon\|\bar{x}\|_{2}^{2}
$$

for some small $\epsilon>0$ and $x^{+}=\mathfrak{f}^{(\cdot)} \in\left\{\mathfrak{f}^{\text {pre }}, \mathfrak{f}^{\text {post }}\right\}$. This approach is guaranteed to have a feasible solution, allows the choice of $\mathfrak{L}$ as a polynomial of arbitrary order, while ensuring that $Q \rightarrow 0$ if the system is dissipative and $\mathfrak{L}$ is a polynomial of suitable order.

For a linear storage-candidate, solving Eq. (17) yields

$$
Q_{1}=\operatorname{diag}\left(0.0014,0.39, \tilde{\epsilon}_{1}, \tilde{\epsilon}_{2}, \tilde{\epsilon}_{3}, \tilde{\epsilon}_{4}\right),
$$

where $\tilde{\epsilon}_{i}<\epsilon$. For a quadratic storage however, we get regularization gains $Q_{2} \prec \epsilon \mathbb{I}_{5}$, indicating that the discrete aircraft system is almost dissipative.

\section{B. Nominal region of attraction}

The MPC recovery controllers do not use thrust; we rely on a nominal flight controller $\kappa_{\mathrm{lvl}}$ to return the aircraft to steady level flight. To ensure safety we switch from the MPC to the nominal control law only once the system state is within the region of attraction of $\kappa_{\mathrm{lvl}}$. As an example we choose the nominal flight trim condition

$$
\begin{aligned}
& x_{\mathrm{lvl}}=\left(13 \mathrm{~m} / \mathrm{s}, 0^{\circ}, 0^{\circ} / \mathrm{s}, 1.35^{\circ},-1.51^{\circ}\right), \\
& u_{\mathrm{lvl}}=\left(0^{\circ} / \mathrm{s}, 0.835 \mathrm{~N}\right),
\end{aligned}
$$

with $\kappa_{\text {lvl }}$ given as continuous LQR feedback for the linearized dynamics around $\left(x_{\mathrm{lvl}}, u_{\mathrm{lvl}}\right)$. The controlled dynamics are given by $\dot{x}=\mathfrak{f}_{\kappa}^{(\cdot)}$.

Using a polynomial Lyapunov-candidate function $\mathfrak{V} \in$ $\mathbb{R}[x]$, we can reformulate (7) into a sum-of-squares optimization problem in order to estimate the region of attraction of $\left(x_{\mathrm{lvl}}, u_{\mathrm{lvl}}\right)$ in nominal flight. An arbitrarily chosen ellipsoidal shape function $\mathfrak{p}$ is used to determine the size of the stable set. As sum-of-squares are limited to non-negativity, we use the relaxed condition

$$
\nabla \mathfrak{V} \cdot \mathfrak{f}_{\kappa}^{(\cdot)}(x) \leq-\epsilon\|\bar{x}\|_{2}^{2}
$$

with $\epsilon>0$ small; let $\Omega_{\mathfrak{V}}^{(\cdot)}$ denote the set of states $x$ such that (21) holds for $\dot{x}=\mathfrak{f}_{\kappa}^{(\cdot)} \in\left\{\mathfrak{f}_{\kappa}^{\text {pre }}, \mathfrak{f}_{\kappa}^{\text {post }}\right\}$. The optimization problem is then given as

$$
\max _{\substack{\lambda, \rho>0 \\
\mathfrak{s}_{0} \in \Sigma[x] \\
\mathbf{s}_{1}, \mathbf{s}_{2} \subset \Sigma[x] \\
\mathfrak{V} \text { p.d. }}} \quad \lambda \text { s.t. }\left\{\begin{array}{r}
\mathfrak{s}_{0} \vdash \Omega_{\mathfrak{p} \leq \rho} \subseteq_{\Sigma} \Omega_{\mathfrak{V} \leq \lambda} \\
\mathbf{s}_{1} \vdash \Omega_{\mathfrak{V} \leq \lambda} \cap \Omega_{\alpha \leq \alpha_{0}} \subseteq_{\Sigma} \Omega_{\mathfrak{V}}^{p r e} \\
\mathbf{s}_{2} \vdash \Omega_{\mathfrak{V} \leq \lambda} \cap \Omega_{\alpha \geq \alpha_{0}} \subseteq_{\Sigma} \Omega_{\mathfrak{V}}^{\text {post }}
\end{array}\right.
$$

which is a bilinear program. We therefore employ the iterative bisection strategy described in [23, 24].

After 49 iterations, the provable stable set in Fig. 1 is obtained with $\lambda^{*}=0.6859$ and $\rho^{*}=28.8696 ; \mathfrak{p}$ is given in the appendix. A larger ROA may be computable using multiple Lyapunov function-candidates $\mathcal{V}^{\text {pre }}, \mathcal{V}^{\text {post }}$.

\section{Numerical Results}

We placed an NMPC controller $\left(\mathrm{NMPC}, \ell_{T}\right)$, a linear MPC (LMPC) controller ${ }^{\pi}$, an EMPC controller with regularization gains $Q_{1}\left(\mathrm{r}-\mathrm{EMPC}, \ell_{R}\right)$ and the unregularized EMPC controller $\left(\mathrm{EMPC}, \ell_{\Delta}\right)$ in closed-loop

ฯ The LMPC controller was designed using the same stage cost and constraints as the NMPC controller but using (5) linearized about the target equilibrium as the prediction model. 

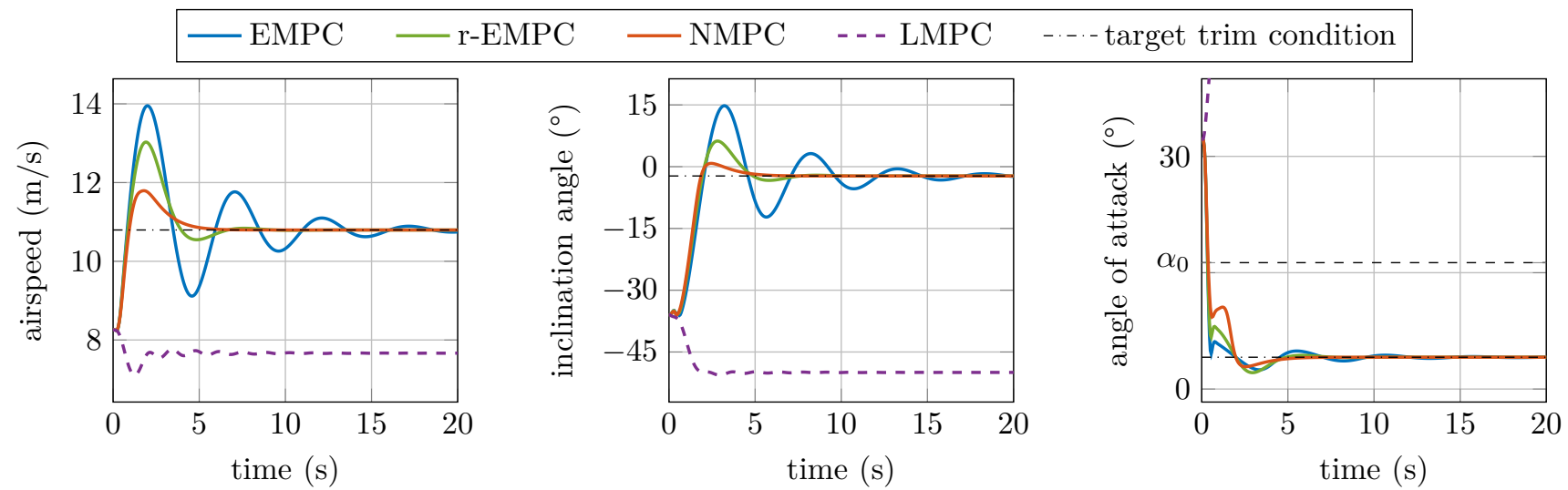

Fig. 2: Economic and tracking MPC for deep-stall recovery in airspeed $V$, inclination $\gamma$, and angle of attack $\alpha$.

with the nonlinear model. The regularization gains $Q_{2}$ are small enough that the closed-loop traces of r-EMPC using $Q_{2}$ were indistinguishable from un-regularized EMPC. All MPC simulations utilize Euler integration with a sampling rate $\tau=50 \mathrm{~ms}$, horizon length $N=120$, and simulation time $T=20 \mathrm{~s}$. The optimal control problem (8) was solved using Ipopt [34]. Further details of the computations are given in the appendix (Tab. I). The initial condition was $\left(8.26 \mathrm{~m} / \mathrm{s},-36.4^{\circ}, 0^{\circ} / \mathrm{s}, 32.6^{\circ},-41^{\circ}\right)$.

Fig. 2 shows the closed-loop trajectories for all four strategies; only LMPC fails to recover the aircraft due to elevator inversion in deep-stall. Of the remaining three, pure EMPC provides the most aggressive approach, partially regaining height by ascent, leading to a slower transition back to normal flight, whereas the regularization gains cause r-EMPC to transition faster. Both economic strategies noticeably overshoot $\|$ the target airspeed and path inclination in order to regain altitude.

Recall that the MPC controllers guide the aircraft to a stable gliding conditions and a nominal flight controller is used to resume level flight. The nominal controller takes over if the state enters its ROA, which was estimated in Section V, (but not earlier than $5 \mathrm{~s}$ into the recovery). The resulting recovery trajectories are shown in Fig. 3. The tracking NMPC recovery shows by far the largest LOA $(8.4 \mathrm{~m})$, while non-regularized and regularized EMPC are similar $(7.5 \mathrm{~m}$ and $7.4 \mathrm{~m})$, with r-EMPC encountering a slightly reduced LOA due to the later switching point of the non-regularized scheme.

\section{CONCLUSION}

Economic MPC is a promising tool for LOA-minimal recovery of an aircraft from upset conditions; however, providing certifiable guarantees of closed-loop stability is nontrivial. In this paper, we applied polynomial SOS programming to a piecewise longitudinal aerodynamics model to prove stability of a deep-stall recovery EMPC strategy, proposed an SOS based regularization scheme

\footnotetext{
\| Overshoot is acceptable in this application except during very low-altitude flight.
}

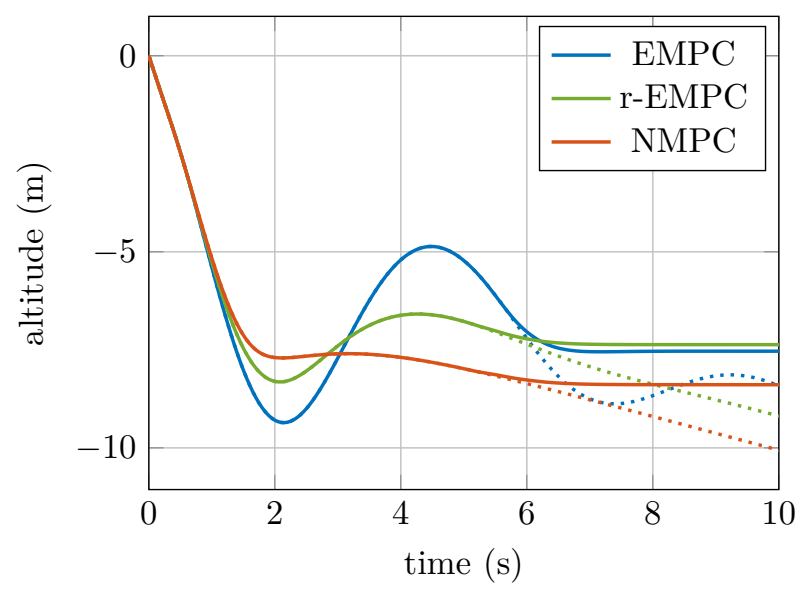

Fig. 3: Upset recovery strategies with switching to nominal control. The full MPC trajectories are shown dotted.

that computes minimal regularization gains needed to ensure dissipativity and illustrated that the gains approach zero as the order of the storage function-candidate is increased. To guarantee stability for all (admissible) states, even very small gains help to avoid undesirable closed-loop behaviour such as periodic oscillations.

Finally, we presented a numerical comparison of different MPC strategies for recovery of nominal flight. Our investigations revealed EMPC offers significant performance advantages compared nonlinear tracking MPC and that regularized EMPC is also compatible with recovery of level flight. Future work includes reduction of computation and application to NASA's GTM.

\section{APPENDIX}

The aircraft states and inputs, were scaled by $d_{V}=$ $10 \mathrm{~m} / \mathrm{s}, d_{\gamma}=45^{\circ}, d_{q}=150^{\circ} / \mathrm{s}, d_{\alpha}=45^{\circ}, d_{\eta}=80^{\circ}$, $d_{d \eta}=100^{\circ} / \mathrm{s}$, and $d_{F}=25 \mathrm{~N}$. The gains for linear and nonlinear tracking MPC were $Q_{x}=\mathbb{I}_{5}$ and $Q_{d \eta}=0.010$ and for nominal LQR feedback, $Q_{x}=\mathbb{I}_{5}$ and $Q_{u}=\mathbb{I}_{2}$ (all gains with respect to the scaled states and inputs).

Eq. (17) for $Q_{2}$ has been solved on a single node of the University of Michigan HPC cluster in $3 \mathrm{~h}$ (Intel Xeon 
TABLE I: Simulation details \& results. Computation time accuracy $\pm 10 \mathrm{~ms}$ (Intel Core i7, $3 \mathrm{GHz}, 16 \mathrm{~GB}$ ).

\begin{tabular}{|c|c|c|c|}
\hline & EMPC & r-EMPC & $\mathrm{NMPC}$ \\
\hline average time $\mathrm{OCP}$ & $1.74 \mathrm{~s}$ & $0.768 \mathrm{~s}$ & $0.674 \mathrm{~s}$ \\
\hline comp. time (MPC) & $951 \mathrm{~s}$ & $503 \mathrm{~s}$ & $486 \mathrm{~s}$ \\
\hline comp. time (to level) & $522 \mathrm{~s}$ & $362 \mathrm{~s}$ & $309 \mathrm{~s}$ \\
\hline$\overline{\text { optimal }} \overline{\operatorname{cost}}(k=\overline{1})^{-}$ & $-{ }^{-} 4 . \overline{7} \overline{6}-$ & $--6 . \overline{6} 5-$ & $-\overline{9 . \overline{8}} \overline{9}^{-}$ \\
\hline residual norm & $<3 \times 10^{-5}$ & $<1 \times 10^{-5}$ & $<9 \times 10^{-4}$ \\
\hline
\end{tabular}

E5, 2.4 GHz, 44 GB). The ellipsoidal shape for Eq. (22) is defined as $\mathfrak{p}=\left(d_{x}^{-1} x\right)^{T} \operatorname{diag}(0.50,0.50,1,1,0.50)\left(d_{x}^{-1} x\right)$; the computation took $6.0 \mathrm{~h}$ on a personal computer (Intel Core i7, $3 \mathrm{GHz}, 16 \mathrm{~GB}$ ). All SOS problems were constructed and solved by sosopt/SeDuMi. As the piecewise model is not differentiable in $\alpha_{0}$ due to Eq. (3), we approximate the aerodynamics by $\mathcal{H}\left(\alpha-\alpha_{0}\right) \mathcal{C}^{\text {pre }}+$ $\left(1-\mathcal{H}\left(\alpha-\alpha_{0}\right)\right) \mathcal{C}^{\text {post }}$, where $\mathcal{H}(\alpha)=\frac{1}{1+e^{-2 \alpha / \mu}}$, and Problem 8 is solved while iteratively decreasing $\mu$. Further details of the simulations are given in Tab. I. The implemented controllers are not yet real-time capable and need efforts to optimize their computational footprint.

\section{REFERENCES}

[1] Loss of Control In-Flight Accident Analysis Report, 2010-2014. Montreal, CA: International Air Transport Association, 2015.

[2] M. H. Smaili, J. Breeman, T. J. J. Lombaerts, J. A. Mulder, Q. P. Chu, and O. Stroosma, "Intelligent Flight Control Systems Evaluation for Loss-of-Control Recovery and Prevention," J. Guid. Control Dynam., vol. 40, no. 4, pp. 890-904, 2016.

[3] N. D. Richards, N. Gandhi, A. J. Bateman, D. H. Klyde, and A. K. Lampton, "Vehicle Upset Detection and Recovery for Onboard Guidance and Control," J. Guid. Control Dynam., vol. 40, no. 4, pp. 920-933, 2017.

[4] R. C. Allen, H. G. Kwatny, and G. Bajpai, "Safe Set Protection and Restoration for Unimpaired and Impaired Aircraft," in AIAA Guidance, Navigation, and Control Conf., Minneapolis, US-MN, 2012.

[5] P. Di Donato, S. Balachandran, K. McDonough, E. Atkins, and I. Kolmanovsky, "Envelope-aware Flight Management for Loss of Control Prevention given Rudder Jam," J. Guid. Control Dynam., vol. 40, pp. 1027-1041, 2017.

[6] B.-C. Chang, H. G. Kwatny, E. R. Ballouz, and D. C. Hartmann, "Aircraft Trim Recovery from Highly Nonlinear Upset Conditions," in AIAA Guidance, Navigation, and Control Conf., San Diego, US-CA, 2016.

[7] H. J. Tol, C. C. de Visser, L. G. Sun, E. van Kampen, and Q. P. Chu, "Multivariate Spline-Based Adaptive Control of High-Performance Aircraft with Aerodynamic Uncertainties," J. Guid. Control Dynam., vol. 39, no. 4, pp. 781-800, 2016.

[8] D. Kim, G. Oh, Y. Seo, and Y. Kim, "Reinforcement LearningBased Optimal Flat Spin Recovery for Unmanned Aerial Vehicle," J. Guid. Control Dynam., vol. 40, no. 4, pp. 1076-1084, 2016.

[9] S. Schuet, T. Lombaerts, J. Kaneshige, K. H. Shish, and V. Stepanyan, "Stall Recovery Guidance Using Fast Model Predictive Control," in AIAA Guidance, Navigation, and Control Conf., Grapevine, US-TX, 2017.

[10] J. J. Engelbrecht and J. A. Engelbrecht, "Optimal Attitude and Flight Vector Recovery for Large Transport Aircraft using Sequential Quadratic Programming," in 2016 Pattern Recognition Association of South Africa and Robotics and Mechatronics International Conf., Stellenbosch, SA, 2016.

[11] D. Sparks and D. Moerder, "Optimal Aircraft Control Upset Recovery With and Without Component Failures," in Proc. of the American Control Conf., Anchorage, US-AK, 2002, pp. 3644-3649.
[12] R. Bunge and I. Kroo, "Automatic Spin Recovery with Minimal Altitude Loss," in 2018 AIAA Guidance, Navigation, and Control Conf., Kissimmee, US-FL, 2018.

[13] M. Ellis, J. Liu, and P. D. Christofides, Economic Model Predictive Control. Basel, CH: Springer, 2017.

[14] T. Cunis, T. Leth, L. C. Totu, and A. la Cour-Harbo, "Identification of Thrust, Lift, and Drag for Deep-stall Flight Data of a Fixed-wing Unmanned Aircraft," in 2018 Int. Conf. on Unmanned Aircraft Systems, Dallas, US-TX, 2018.

[15] T. Cunis, J.-P. Condomines, L. Burlion, and A. la Cour-Harbo, "Dynamic Stability Analysis of Aircraft Flight in Deep-stall," Under review for J. Aircraft, 2019.

[16] U. Eren, A. Prach, B. B. Koçer, S. V. Raković, E. Kayacan, and B. Açlkmeşe, "Model Predictive Control in Aerospace Systems: Current State and Opportunities," J. Guid. Control Dynam., vol. 40, no. 7, pp. 1541-1566, 2017.

[17] D. Angeli, R. Amrit, and J. B. Rawlings, "On Average Performance and Stability of Economic Model Predictive Control," IEEE Trans. Automat. Contr., vol. 57, no. 7, pp. 1615-1626, 2012.

[18] M. Zanon and T. Faulwasser, "Economic MPC without terminal constraints: Gradient-correcting end penalties enforce asymptotic stability," J. of Process Contr., vol. 63, pp. 1-14, 2018.

[19] J. Jäschke, X. Yang, and L. T. Biegler, "Fast economic model predictive control based on NLP-sensitivities," J. Process Contr., vol. 24, no. 8, pp. 1260-1272, 2014.

[20] P. A. Parillo, "Semidefinite programming relaxations for semialgebraic problems," Math. Program. B, vol. 96, no. 2, pp. 293-320, 2003.

[21] W. Tan, "Nonlinear Control Analysis and Synthesis using Sum-of-Squares Programming," PhD thesis, UC Berkeley, Berkeley, US-CA, 2006.

[22] P. Seiler and G. J. Balas, "Quasiconvex sum-of-squares programming," in 49th IEEE Conf. on Decision and Control, Atlanta, US-GA, 2010, pp. 3337-3342.

[23] U. Topcu, A. Packard, and P. Seiler, "Local stability analysis using simulations and sum-of-squares programming," Automatica, vol. 44, no. 10, pp. 2669-2675, 2008 .

[24] A. Chakraborty, P. Seiler, and G. J. Balas, "Nonlinear region of attraction analysis for flight control verification and validation," Control Eng. Pract., vol. 19, no. 4, pp. 335-345, 2011.

[25] A. A. Ahmadi and P. A. Parrilo, "Non-monotonic Lyapunov functions for stability of discrete time nonlinear and switched systems," in 47th IEEE Conf. on Decision and Control, 2008, pp. 614-621.

[26] G. Chesi, "Establishing robust stability of discrete-time systems with time-varying uncertainty: The Gram-SOS approach," Automatica, vol. 50, no. 11, pp. 2813-2821, 2014.

[27] C. Ebenbauer and F. Allgöwer, "Analysis and design of polynomial control systems using dissipation inequalities and sum of squares," Comp. Chem. Eng., vol. 30, pp. 1590-1602, 2006.

[28] T. Faulwasser, M. Korda, C. N. Jones, and D. Bonvin, "Turnpike and dissipativity properties in dynamic real-time optimization and economic MPC," in 53rd IEEE Conf. on Decision and Control, Los Angeles, US-CA, 2014, pp. 2734-2739.

[29] M. Korda and C. N. Jones, "Automatica Stability and performance verification of optimization-based," Automatica, vol. 78, pp. 34-45, 2017.

[30] "Airplane Flying Handbook," Flight Standards Service, Washington, US-DC, FAA handbook FAA-H-8083-3B, 2016.

[31] T. Cunis and A. la Cour-Harbo, "Piecewise Polynomial Model of the Aerodynamic Coefficients of the Cumulus One Unmanned Aircraft," Sky-Watch A/S, Støvring, DK, Tech. Rep. hal-02280789. [Online]. Available: https://hal-enac. archives-ouvertes.fr/hal-02280789

[32] J. P. La Salle, "Some Extensions of Liapunov's Second Method," IRE Trans. Circuit Theory, vol. CT-7, pp. 520-527, $\operatorname{dec} 1960$.

[33] L. Grüne and J. Pannek, Nonlinear Model Predictive Control: Theory and Algorithms, 2nd ed. Basel, CH: Springer, 2017.

[34] A. Wächter and L. T. Biegler, "On the implementation of an interior-point filter line-search algorithm for large-scale nonlinear programming," Math. Program. A, vol. 106, pp. 25-57, 2006. 